\title{
Fatalism and stigma amongst cancer patients in south western Punjab
}

\author{
Lavjit Kaur 冈 \\ Punjab Agricultural University, Ludhiana, Punjab, India. \\ Shalini Sharma \\ Punjab Agricultural University, Ludhiana, Punjab, India. \\ Amanpreet Kaur \\ Punjab Agricultural University, Ludhiana, Punjab, India.
}

\begin{tabular}{|c|c|}
\hline ARTICLE INFO & ABSTRACT \\
\hline $\begin{array}{l}\text { Received : } 06 \text { August } 2021 \\
\text { Revised : } 24 \text { September } 2021 \\
\text { Accepted : } 05 \text { October } 2021 \\
\text { Available online: } 19 \text { December } 2021 \\
\text { Key Words: } \\
\text { Beliefs } \\
\text { Taboo } \\
\text { Traditional Knowledge } \\
\text { Fatalism } \\
\text { Diagnosis }\end{array}$ & $\begin{array}{l}\text { The ideology and beliefs have a profound impact upon once lifestyle. Economic } \\
\text { status, level of knowledge and awareness, beliefs and notions, social support } \\
\text { system available, constitutes important determinants impacting to copeup } \\
\text { strategies for cancer victims. The present study was conducted to assess the } \\
\text { fatalism and stigma amongst cancer patients in south western Punjab. Two } \\
\text { districts i.e. Bathinda and Sri Muktsar Sahib were randomly selected. Majority } \\
\text { (87.5\%) of the respondents believe that in cancer patients attributed cancer has } \\
\text { caused due to their karmas. Forty six per cent of the respondents believed that } \\
\text { cancer is contagious. One third (34.3\%) of the respondents and their family } \\
\text { members used the term 'cancer' as they believed that taking the name of } \\
\text { disease had nothing to do with its curability versus non-curability or to describe } \\
\text { the dreaded disease from which they were suffering. One third of the male } \\
\text { respondents perceived imbalanced diet and sedentary lifestyle behind as a } \\
\text { reasoning causing cancer and half of the male respondents and significantly } \\
\text { majority of the female respondents (93.3\%) perceived substance abuse as a } \\
\text { reason behind causing cancer in the region. The study suggests that NGOs, } \\
\text { religious bodies and philanthropists can contribute significantly towards } \\
\text { treatment, management and rehabilitation of cancer victims. The use of } \\
\text { recommended doses of pesticides/ insecticides and fertilizers and } \\
\text { unprecedented use of chemicals should be monitored through government } \\
\text { agencies and guilty should be punished. }\end{array}$ \\
\hline
\end{tabular}

\section{Introduction}

Cancer has long been one of the most feared beginning with prevention and proceeding to diseases, widely regarded to be synonymous with palliation - a challenging task. The ideology and death (Robb et al., 2014; Katz et al., 1987; beliefs have a profound impact upon once lifestyle. Corrigan, 2014; Daher, 2012; Knapp et al., 2014). Economic status, level of knowledge and In India, the annual burden for new cancers is awareness, beliefs and notions, social support approximately one million, and the mortality rate is system available, constitutes important 67.2 per 1, 00,000 (WHO, 2012) which is primarily determinants impacting coping up strategies for the result of late diagnosis. Lack of awareness fuels cancer victims. Role of beliefs and ideologies in many myths and misconceptions related to cancer, determining the cancer variations across different which perpetuates the stigma associated with it regions have been discussed and debated well (Rob et al., 2014; Rai et al., 2014; Pahwa et al., (Kaur and Sharma, 2020; Kaur, 2015). These also 2005). This stigma influences the way people reflect the level of knowledge regarding various perceive cancer, which renders cancer control- facets of life. However delays may occur at

Corresponding author E-mail: lavjit-aes@pau.edu

Doi: https://doi.org/10.36953/ECJ.2021.22343

This work is licensed under Attribution-Non Commercial 4.0 International (CC BY-NC 4.0)

(C) ASEA 
different stages of the cancer diagnostic journey and have been commonly defined as being either patient focused or healthcare provider focused. Commonly, delay is found further categorized into different component delays such as patient delay, health care provider delay, referral delay and system delay. Calculation of the total economic burden of a particular cancer or cancer in general, provides information to decision makers for mobilizing political and financial support for cancer care and research (Kaur and Sharma, 2019). Since resources are not unlimited and costs do matter delay, referral delay and system delay. Delay in seeking medical care by cancer victims might result in disastrous outcomes costing even the life, as cancer cells multiply too fast and affect badly all the major organs of the human being (Chatat et al., 2015; Kaur, 2013; Kishore et al., 2007; Kaur, 2015; Kaur and Sharma 2021).

\section{Material and Methods}

The present study was conducted in cotton belt of Punjab which constitute south western districts viz. Bathinda, Sri Muktsar Sahib, Ferozepur, Mansa, Faridkot, Sangrur, Fazilka and Barnala of Punjab during 2017-2018. Two districts i.e. Bathinda and Sri Muktsar Sahib (hereafter, Sri Muktsar Sahib will be referred as Muktsar in the text) were randomly selected. At the second stage, two blocks were randomly selected from each selected district to obtain a total of four blocks. At third stage, three villages from each block were taken for the purpose of investigation. So, the present study was conducted in twelve villages randomly selected from south western region of Punjab. After the selection of the villages, a list of all the persons suffering from cancer or had cancer (though recovered later on) during the period of five years preceding the study comprised the sample for study. The present study was based on the primary data procured on the basis of records maintained by the hospitals both public and private, Auxiliary Nurse Midwives (ANMs), chemists and sarpanches. Change agents were also contacted for procuring data. At the final stage, 160 cancer patients were personally interviewed for the study. The data were tabulated and analyzed by using appropriate statistical tools. Thus, frequency distribution, percentages and Chi-square test were used to reach the logical conclusion.

\section{Chi Square}

Chi square test was applied to check the association between socio-economic indicators and anxiety level of cancer patients. The statistical equation is given below:

Where,

$$
(x)^{2}=\in \frac{(o-e)^{2}}{e}
$$

$$
\begin{array}{ll}
x 2= & \text { Chi-square value } \\
\mathrm{O}= & \text { Observed Frequency }
\end{array}
$$

$\mathrm{E}=$ Expected Frequency assigned as equal frequency to all the numbers by dividing the total sum of frequency to each number.

\section{$\Sigma=$ Summation}

\section{Results and Discussion}

\section{Beliefs and perceptions regarding cancer}

It is seen that role of beliefs and ideologies in determining the cancer variations across different regions have been discussed and debated well. Health seeking behavior is pertinent in determining the health status, which in turn is outcome of deeprooted notions and beliefs held by a person or community. These beliefs may either result in prompt action or may cause delay in action by the person concerned. Delay in seeking medical care by cancer victims might result in disastrous outcomes costing even the life, as cancer cells multiply too fast and affect badly all the major organs of the human being. So, it is pertinent to correct false notions and unscientific beliefs held by the society in order to save upon the precious human resource which might get wasted due to dilly dallying the actions required to mitigate the situation. Various beliefs and perceptions directly or indirectly affecting cancer management and mitigation are discussed in the following section.

\section{Belief in Karma}

Karma is a Sanskrit word from the root 'Kri', meaning 'to do', karma literally means 'that which is done' or 'action', but is also a philosophical doctrine. In simple term-, it is the law of cause and effect, the principle that 'as you sow, so shall you reap'. The word also refers to the totality of mankind's actions and their concomitant reactions in current and previous lives, all of which determines the future (Rangekar, 2012). The concept of karma is linked to the concept of reincarnation, in that the effect of actions can last through several lifetimes. Karma can be modified 
or changed through right action, and a state beyond the effects of karma is achieved through union with the divine. The karma is understood as that which causes the entire cycle of cause and effect originating in ancient India and treated in Hindu, Jain, Sikh and Buddhist philosophies (Rangekar, 2012).The perusal of the table 1 depicts the distribution of respondents on the basis of their believe in karma. It has been revealed from the study that majority $(87.5 \%)$ of the respondents believe that cancer patients attributed cancer has caused due to their karmas. The attribution of cancer to karma by the respondents and search for meaning behind the disease may be termed as "religious purification" which means confession of sins and asking for forgiveness. It is also confirmed that, the role of karma in attribution of meanings to the disease (Pargement, 1997). Only 12.50 percent of the respondents didn't accord any role to their karmas for their disease.

\section{Belief regarding contagiousness of cancer}

Contagious means that cancer spreads from one person to another through direct or indirect contact. Cancer is not a contagious disease that could spreads from person to person. The only situation in which cancer can spread from one person to another is in the case of organ or tissue transplantation. A person who receives an organ or tissue from a donor who had cancer in the past may be at increased risk of developing a transplantrelated cancer in the future. Due to fear that cancer spreads from one person to another led some of the cancer patients to worry about the health of their family members who were constantly in contact with them. The study observed widespread of misgiving regarding contagiousness of the disease as shown in Table 2. Forty-six per cent of the respondents believed that cancer is contagious. Most of the respondents who believed that cancer is contagious were illiterate.

\section{Common nomenclature used for cancer}

The fear of certain diseases especially cancer (which earlier was considered as non curable), that even taking name is considered dangerous. Off late cancer has become so widespread in the study area, that it has even altered stigmas and severe motions attached with it. The perusal of Table 3 highlighted the common nomenclature used for cancer by the respondents. Earlier people avoid using the word cancer to portray the disease to the other individual in the society. But now as individuals are more aware about the disease and don't hesitate to take it. One third $(34.3 \%)$ of the respondents and their family members used the term 'cancer' as they believed that taking the name of disease had nothing to do with its curability versus noncurability or to describe the dreaded disease from which they were suffering. The second most common word used by the respondents $(30.0 \%)$ was duji bimari followed by gand $(10.0 \%)$, chandri bimari $(8.1 \%)$, opri bimari (8.1\%), Bikaner wali bimari $(8.1 \%)$ and ohi wali bimari $(1.2 \%)$ in the study region of Punjab. The common word ohiwalibimari was used by only one male respondent of Bathinda district and one female respondent of the Muktsar district respectively.

\section{Beliefs regarding mitigating practices}

The perusal of Table 4 highlights the religious believe regarding various mitigating practices of the cancer patients. In south western region of Punjab, majority (76.8\%) of the respondents believed in sukhna. Sukhna comprised various forms of commitments made by a person one's his/her wish gets fulfilled. It included reciting Sukhmani Sahib Paths and other charity works. Three fourth $(71.8 \%)$ of the respondents disclosed that devastation caused by cancer increased faith in God after the diagnosis of the disease. Twentythree per cent of the respondents took dip in holy water at holy places in order to seek god's blessings and wade off evil spirits/eye. It was revealed by the respondents that they did itto get rid of their past sins. Respondents (17.5\%) were observed visiting Gurudwaras on special days like Amavasya, Gurupurav, Punnia etc. followed by reciting the path daily (13.1\%), regular visit to gurudwara sahib $(9.3 \%)$, practicing chaliya $(4.3 \%)$. Chaliya is the practice of visiting holy places uninterrupted for 40 days at a stretch. Two respondents offer the degh at Dera Sacha Saudha with the conviction of mitigating the disease.

\section{Belief in being secretive about cancer}

Due to the stigma attached with the disease, people were often hesitates to share about the prevalence and were secretive about their disease. The data in table 5 highlights that two third $(62.5 \%)$ of the respondents did not hesitate to share about their disease. They shared about their disease with others 
Table 1: Distribution of respondents on the basis of belief in association of cancer with karma

\begin{tabular}{|c|c|c|c|c|c|c|c|c|c|}
\hline \multirow{2}{*}{$\begin{array}{l}\text { Believe in } \\
\text { Karma }\end{array}$} & \multicolumn{3}{|c|}{$\begin{array}{c}\text { Bathinda } \\
(n=76)\end{array}$} & \multicolumn{3}{|c|}{$\begin{array}{c}\text { Sri Muktsar Sahib } \\
(\mathrm{n}=84)\end{array}$} & \multicolumn{3}{|c|}{$\begin{array}{c}\text { South Western Region } \\
(n=160)\end{array}$} \\
\hline & $\begin{array}{c}\text { Male } \\
(\mathbf{n}=\mathbf{2 2})\end{array}$ & $\begin{array}{c}\text { Female } \\
(\mathrm{n}=54)\end{array}$ & $\begin{array}{r}\text { Total } \\
(n=76)\end{array}$ & $\begin{array}{c}\text { Male } \\
(\mathrm{n}=\mathbf{3 4})\end{array}$ & $\begin{array}{c}\text { Female } \\
(\mathbf{n}=\mathbf{5 0})\end{array}$ & $\begin{array}{r}\text { Total } \\
(\mathrm{n}=\mathbf{8 4})\end{array}$ & $\begin{array}{c}\text { Male } \\
(\mathrm{n}=56)\end{array}$ & $\begin{array}{l}\text { Female } \\
(n=104)\end{array}$ & $\begin{array}{c}\text { Total } \\
(\mathrm{n}=\mathbf{1 6 0})\end{array}$ \\
\hline Yes & $\begin{array}{c}16 \\
(72.72) \\
\end{array}$ & $\begin{array}{c}49 \\
(90.74) \\
\end{array}$ & $\begin{array}{c}65 \\
(85.52) \\
\end{array}$ & $\begin{array}{c}29 \\
(85.29) \\
\end{array}$ & $\begin{array}{c}46 \\
(92.00)\end{array}$ & $\begin{array}{c}75 \\
(89.28)\end{array}$ & $\begin{array}{c}45 \\
(80.35)\end{array}$ & $\begin{array}{c}95 \\
(91.34)\end{array}$ & $\begin{array}{c}140 \\
(87.50)\end{array}$ \\
\hline No & $\begin{array}{c}6 \\
(27.27) \\
\end{array}$ & $\begin{array}{c}5 \\
(9.25)\end{array}$ & $\begin{array}{c}11 \\
(14.47)\end{array}$ & $\begin{array}{c}5 \\
(17.24)\end{array}$ & $\begin{array}{c}4 \\
(8.00)\end{array}$ & $\begin{array}{c}9 \\
(10.71)\end{array}$ & $\begin{array}{c}11 \\
(19.64)\end{array}$ & $\begin{array}{c}9 \\
(8.65)\end{array}$ & $\begin{array}{c}20 \\
(12.50)\end{array}$ \\
\hline
\end{tabular}

Note: Figures in the parentheses indicate percentage

Table 2: Distribution of respondents on the basis of belief regarding contagiousness of cancer

\begin{tabular}{|c|c|c|c|c|c|c|c|c|c|}
\hline \multirow{2}{*}{ Particulars } & \multicolumn{3}{|c|}{$\begin{array}{c}\text { Bathinda } \\
(n=76)\end{array}$} & \multicolumn{3}{|c|}{ Sri Muktsar Sahib $(n=84)$} & \multicolumn{3}{|c|}{$\begin{array}{c}\text { South Western Region } \\
(n=160)\end{array}$} \\
\hline & $\begin{array}{c}\text { Male } \\
(\mathrm{n}=\mathbf{2 2})\end{array}$ & $\begin{array}{c}\text { Female } \\
(n=54)\end{array}$ & $\begin{array}{c}\text { Total } \\
(n=76)\end{array}$ & $\begin{array}{c}\text { Male } \\
(\mathrm{n}=34)\end{array}$ & $\begin{array}{c}\text { Female } \\
(\mathrm{n}=50)\end{array}$ & $\begin{array}{c}\text { Total } \\
(\mathrm{n}=84)\end{array}$ & $\begin{array}{c}\text { Male } \\
(\mathrm{n}=56)\end{array}$ & $\begin{array}{l}\text { Female } \\
(n=104)\end{array}$ & $\begin{array}{c}\text { Total } \\
(n=160)\end{array}$ \\
\hline Cont: & $\begin{array}{c}10 \\
(45.45)\end{array}$ & $\begin{array}{c}27 \\
(50.00)\end{array}$ & $\begin{array}{c}37 \\
(48.68)\end{array}$ & $\begin{array}{c}10 \\
(29.41)\end{array}$ & $\begin{array}{c}23 \\
(46.00)\end{array}$ & $\begin{array}{c}33 \\
(39.28)\end{array}$ & $\begin{array}{c}20 \\
(35.71)\end{array}$ & $\begin{array}{c}50 \\
(48.07)\end{array}$ & $\begin{array}{c}70 \\
(43.75)\end{array}$ \\
\hline $\begin{array}{l}\text { Non } \\
\text { Contagious }\end{array}$ & $\begin{array}{c}12 \\
(54.55)\end{array}$ & $\begin{array}{c}27 \\
(50.00)\end{array}$ & $\begin{array}{c}39 \\
(51.31)\end{array}$ & $\begin{array}{c}24 \\
(70.58)\end{array}$ & $\begin{array}{c}27 \\
(54.00)\end{array}$ & $\begin{array}{c}51 \\
(60.71)\end{array}$ & $\begin{array}{c}36 \\
(64.28)\end{array}$ & $\begin{array}{c}54 \\
(51.92)\end{array}$ & $\begin{array}{c}90 \\
(56.25)\end{array}$ \\
\hline
\end{tabular}

Note: Figures in the parentheses indicate percentage

Table 3: Distribution of respondents on the basis of common nomenclature used for cancer

\begin{tabular}{|c|c|c|c|c|c|c|c|c|c|}
\hline \multirow{2}{*}{$\begin{array}{l}\text { Common } \\
\text { nomenclature } \\
\text { used for cancer }\end{array}$} & \multicolumn{3}{|c|}{$\begin{array}{c}\text { Bathinda } \\
(\mathrm{n} 1=76)\end{array}$} & \multicolumn{3}{|c|}{ Sri Muktsar Sahib (n2=84) } & \multicolumn{3}{|c|}{$\begin{array}{l}\text { South Western Region } \\
(\mathrm{N}=160)\end{array}$} \\
\hline & $\begin{array}{c}\text { Male } \\
(\mathrm{n}=\mathbf{2 2})\end{array}$ & $\begin{array}{c}\text { Female } \\
(n=54)\end{array}$ & $\begin{array}{c}\text { Total } \\
(\mathrm{n}=76)\end{array}$ & $\begin{array}{c}\text { Male } \\
(n=34)\end{array}$ & $\begin{array}{c}\text { Female } \\
(\mathbf{n}=50)\end{array}$ & $\begin{array}{c}\text { Total } \\
(n=84)\end{array}$ & $\begin{array}{c}\text { Male } \\
(n=56)\end{array}$ & $\begin{array}{l}\text { Female } \\
(n=104)\end{array}$ & $\begin{array}{c}\text { Total } \\
(\mathrm{n}=\mathbf{1 6 0})\end{array}$ \\
\hline Cancer & $\begin{array}{c}9 \\
(40.91)\end{array}$ & $\begin{array}{c}16 \\
(29.63)\end{array}$ & $\begin{array}{c}25 \\
(32.89)\end{array}$ & $\begin{array}{c}14 \\
(41.18)\end{array}$ & $\begin{array}{c}16 \\
(32.00)\end{array}$ & $\begin{array}{c}30 \\
(35.71)\end{array}$ & $\begin{array}{c}23 \\
(41.07)\end{array}$ & $\begin{array}{c}32 \\
(30.76)\end{array}$ & $\begin{array}{c}55 \\
(34.37)\end{array}$ \\
\hline $\begin{array}{ll}\text { Ohi } & \text { Wali } \\
\text { Bimari } & \end{array}$ & - & $\begin{array}{c}1 \\
(1.85)\end{array}$ & $\begin{array}{c}1 \\
(1.32)\end{array}$ & $\begin{array}{c}1 \\
(2.94)\end{array}$ & - & $\begin{array}{c}1 \\
(1.19)\end{array}$ & $\begin{array}{c}1 \\
(1.78)\end{array}$ & $\begin{array}{c}1 \\
(0.96)\end{array}$ & $\begin{array}{c}2 \\
(1.25)\end{array}$ \\
\hline Duji Bimari & $\begin{array}{c}7 \\
(31.82)\end{array}$ & $\begin{array}{c}14 \\
(25.93)\end{array}$ & $\begin{array}{c}21 \\
(27.63)\end{array}$ & $\begin{array}{c}10 \\
(29.41)\end{array}$ & $\begin{array}{c}17 \\
(34.00)\end{array}$ & $\begin{array}{c}27 \\
(32.14)\end{array}$ & $\begin{array}{c}17 \\
(30.35)\end{array}$ & $\begin{array}{c}31 \\
(29.80)\end{array}$ & $\begin{array}{c}48 \\
(30.00)\end{array}$ \\
\hline Opri Bimari & $\begin{array}{c}1 \\
(4.55)\end{array}$ & $\begin{array}{c}5 \\
(9.26)\end{array}$ & $\begin{array}{c}6 \\
(7.89)\end{array}$ & $\begin{array}{c}3 \\
(8.82)\end{array}$ & $\begin{array}{c}4 \\
(8.00)\end{array}$ & $\begin{array}{c}7 \\
(8.33)\end{array}$ & $\begin{array}{c}4 \\
(7.14)\end{array}$ & $\begin{array}{c}9 \\
(8.65)\end{array}$ & $\begin{array}{c}13 \\
(8.12)\end{array}$ \\
\hline $\begin{array}{ll}\text { Bikaner } & \text { Wali } \\
\text { Bimari } & \end{array}$ & - & $\begin{array}{c}5 \\
(9.26)\end{array}$ & $\begin{array}{c}5 \\
(6.58)\end{array}$ & $\begin{array}{c}2 \\
(5.88)\end{array}$ & $\begin{array}{c}6 \\
(12.00)\end{array}$ & $\begin{array}{c}8 \\
(9.52)\end{array}$ & $\begin{array}{c}2 \\
(3.57)\end{array}$ & $\begin{array}{c}11 \\
(10.57)\end{array}$ & $\begin{array}{c}13 \\
(8.12)\end{array}$ \\
\hline Chandri Bimari & $\begin{array}{c}3 \\
(13.64)\end{array}$ & $\begin{array}{c}6 \\
(11.11) \\
\end{array}$ & $\begin{array}{c}9 \\
(11.84)\end{array}$ & $\begin{array}{c}2 \\
(5.88) \\
\end{array}$ & $\begin{array}{c}2 \\
(4.00) \\
\end{array}$ & $\begin{array}{c}4 \\
(4.76) \\
\end{array}$ & $\begin{array}{c}5 \\
(8.92)\end{array}$ & $\begin{array}{c}8 \\
(7.69) \\
\end{array}$ & $\begin{array}{c}13 \\
(8.12)\end{array}$ \\
\hline Gand & $\begin{array}{c}2 \\
(9.09)\end{array}$ & $\begin{array}{c}7 \\
(12.96)\end{array}$ & $\begin{array}{c}9 \\
(11.84)\end{array}$ & $\begin{array}{c}2 \\
(5.88)\end{array}$ & $\begin{array}{c}5 \\
(10.00)\end{array}$ & $\begin{array}{c}7 \\
(8.33)\end{array}$ & $\begin{array}{c}4 \\
(7.14)\end{array}$ & $\begin{array}{c}12 \\
(11.53)\end{array}$ & $\begin{array}{c}16 \\
(10.00)\end{array}$ \\
\hline
\end{tabular}

Note: Figures in the parentheses indicate percentage

in order to get more and more information ghar cancer aa" in the village. For instance, in regarding treatment, management and precautions village Jajjal in early 90's the matrimonial alliances etc. about the disease. But 37.5 percent of the were struck off due to cancer disease.

respondents still hesitant to share about the disease and kept it secret from others due to social stigma as they thought it could also effects on matrimonial prospects of their sons and the daughters, if people know about it they will start discriminating the min one or the other way, people will start recognizing our home by the name of "oh wala ghar jihna de Overall, females from both the district hesitated more than males to share were secretive about the disease in the region.

Belief regarding the impact of cancer

A fatalistic view regarding cancer is an important force that discourages a patient from leading productive life and propagates a sense of fear about 
Table 4: Distribution of respondents on the basis of beliefs regarding mitigation practices (Multiple responses).

\begin{tabular}{|c|c|c|c|c|c|c|c|c|c|}
\hline \multirow[t]{2}{*}{ Practices } & \multicolumn{3}{|c|}{$\begin{array}{c}\text { Bathinda } \\
(\mathrm{n} 1=76)\end{array}$} & \multicolumn{3}{|c|}{ Sri Muktsar Sahib (n2=84) } & \multicolumn{3}{|c|}{$\begin{array}{c}\text { South Western Region } \\
(\mathrm{N}=160)\end{array}$} \\
\hline & $\begin{array}{c}\text { Male } \\
(\mathrm{n}=\mathbf{2 2})\end{array}$ & $\begin{array}{c}\text { Female } \\
(\mathrm{n}=54)\end{array}$ & $\begin{array}{c}\text { Total } \\
(n=76)\end{array}$ & $\begin{array}{c}\text { Male } \\
(\mathrm{n}=34)\end{array}$ & $\begin{array}{c}\text { Female } \\
(n=50)\end{array}$ & $\begin{array}{r}\text { Total } \\
(\mathrm{n}=84)\end{array}$ & $\begin{array}{c}\text { Male } \\
(\mathrm{n}=56)\end{array}$ & $\begin{array}{l}\text { Female } \\
(n=104)\end{array}$ & $\begin{array}{c}\text { Total } \\
(\mathrm{n}=160)\end{array}$ \\
\hline Sukhna & $\begin{array}{c}12 \\
(54.54)\end{array}$ & $\begin{array}{c}40 \\
(71.42) \\
\end{array}$ & $\begin{array}{c}52 \\
(68.42) \\
\end{array}$ & $\begin{array}{c}29 \\
(85.29) \\
\end{array}$ & $\begin{array}{c}42 \\
(84.00) \\
\end{array}$ & $\begin{array}{c}71 \\
(84.52) \\
\end{array}$ & $\begin{array}{c}41 \\
(73.21) \\
\end{array}$ & $\begin{array}{c}82 \\
(78.84) \\
\end{array}$ & $\begin{array}{c}123 \\
(76.87) \\
\end{array}$ \\
\hline $\begin{array}{l}\text { Regular visit } \\
\text { to gurudwara } \\
\text { sahib }\end{array}$ & $\begin{array}{c}2 \\
(9.09)\end{array}$ & $\begin{array}{c}2 \\
(3.70)\end{array}$ & $\begin{array}{c}4 \\
(5.26)\end{array}$ & $\begin{array}{c}5 \\
(14.70)\end{array}$ & $\begin{array}{c}6 \\
(7.14)\end{array}$ & $\begin{array}{c}11 \\
(13.09)\end{array}$ & $\begin{array}{c}7 \\
(12.5)\end{array}$ & $\begin{array}{c}8 \\
(7.69)\end{array}$ & $\begin{array}{l}15 \\
(9.37)\end{array}$ \\
\hline $\begin{array}{l}\text { Chaliya } \\
\text { days })\end{array}$ & $\begin{array}{c}1 \\
(4.54)\end{array}$ & $\begin{array}{c}3 \\
(5.55)\end{array}$ & $\begin{array}{c}4 \\
(5.26)\end{array}$ & $\begin{array}{c}1 \\
(2.94)\end{array}$ & $\begin{array}{c}2 \\
(4.00)\end{array}$ & $\begin{array}{c}3 \\
(3.57)\end{array}$ & $\begin{array}{c}2 \\
(3.57)\end{array}$ & $\begin{array}{c}5 \\
(4.80)\end{array}$ & $\begin{array}{c}7 \\
(4.37)\end{array}$ \\
\hline $\begin{array}{l}\text { Reciting Path } \\
\text { daily }\end{array}$ & $\begin{array}{c}6 \\
(27.27)\end{array}$ & $\begin{array}{c}2 \\
(3.70)\end{array}$ & $\begin{array}{c}8 \\
(10.52)\end{array}$ & $\begin{array}{c}13 \\
(38.23)\end{array}$ & - & $\begin{array}{c}13 \\
(15.47)\end{array}$ & $\begin{array}{c}19 \\
(33.92)\end{array}$ & $\begin{array}{c}2 \\
(1.92)\end{array}$ & $\begin{array}{c}21 \\
(13.12)\end{array}$ \\
\hline $\begin{array}{l}\text { Taking dip at } \\
\text { holy places }\end{array}$ & $\begin{array}{c}4 \\
(18.18)\end{array}$ & $\begin{array}{c}15 \\
(27.77)\end{array}$ & $\begin{array}{c}19 \\
(25.00)\end{array}$ & $\begin{array}{c}10 \\
(29.41)\end{array}$ & $\begin{array}{c}9 \\
(18.00)\end{array}$ & $\begin{array}{c}19 \\
(22.61)\end{array}$ & $\begin{array}{c}14 \\
(25.00)\end{array}$ & $\begin{array}{c}24 \\
(23.07)\end{array}$ & $\begin{array}{c}38 \\
(23.75)\end{array}$ \\
\hline $\begin{array}{l}\text { Offering } \\
\text { Degh }\end{array}$ & $\begin{array}{c}1 \\
(4.54)\end{array}$ & $\begin{array}{c}1 \\
(1.85)\end{array}$ & $\begin{array}{c}2 \\
(2.63)\end{array}$ & - & - & - & $\begin{array}{c}1 \\
(1.78)\end{array}$ & $\begin{array}{c}1 \\
(0.96)\end{array}$ & $\begin{array}{c}2 \\
(1.25)\end{array}$ \\
\hline $\begin{array}{l}\text { Visiting on } \\
\text { special days } \\
\text { to } \\
\text { gurudwara* }\end{array}$ & $\begin{array}{c}6 \\
(27.27)\end{array}$ & $\begin{array}{c}5 \\
(9.25)\end{array}$ & $\begin{array}{c}11 \\
(14.47)\end{array}$ & $\begin{array}{c}7 \\
(20.58)\end{array}$ & $\begin{array}{c}10 \\
(20.00)\end{array}$ & $\begin{array}{c}17 \\
(20.23)\end{array}$ & $\begin{array}{c}13 \\
(23.21)\end{array}$ & $\begin{array}{c}15 \\
(14.42)\end{array}$ & $\begin{array}{c}28 \\
(17.50)\end{array}$ \\
\hline
\end{tabular}

Note: Figures in the parentheses indicate percentage

*on Amavasya, Gurupurav, Punnia

Table 5: Distribution of the respondents on the basis of belief being secretive about cancer.

\begin{tabular}{|l|c|c|c|c|c|c|c|c|c|}
\hline \multirow{2}{*}{$\begin{array}{l}\text { Secretive } \\
\text { about cancer }\end{array}$} & \multicolumn{3}{|c|}{ Bathinda(n=76) } & \multicolumn{3}{c|}{$\begin{array}{c}\text { Sri Muktsar Sahib } \\
(\mathbf{n}=\mathbf{8 4})\end{array}$} & \multicolumn{3}{c|}{$\begin{array}{c}\text { South Western } \\
\text { Region(n=160) }\end{array}$} \\
\cline { 2 - 10 } & $\begin{array}{c}\text { Male } \\
(\mathbf{n = 2 2})\end{array}$ & $\begin{array}{c}\text { Female } \\
(\mathbf{n}=\mathbf{5 4})\end{array}$ & $\begin{array}{c}\text { Total } \\
(\mathbf{n = 7 6 )}\end{array}$ & $\begin{array}{c}\text { Male } \\
(\mathbf{n = 3 4 )}\end{array}$ & $\begin{array}{c}\text { Female } \\
(\mathbf{n = 5 0 )}\end{array}$ & $\begin{array}{c}\text { Total } \\
(\mathbf{n = 8 4})\end{array}$ & $\begin{array}{c}\text { Male } \\
(\mathbf{n}=\mathbf{5 6})\end{array}$ & $\begin{array}{c}\text { Female } \\
(\mathbf{n = 1 0 4})\end{array}$ & $\begin{array}{c}\text { Total } \\
(\mathbf{n}=\mathbf{1 6 0})\end{array}$ \\
\hline Yes & 6 & 21 & 27 & 11 & 22 & 33 & 17 & 43 & 60 \\
& $(27.27)$ & $(38.89)$ & $(35.52)$ & $(32.35)$ & $(44.00)$ & $(39.29)$ & $(30.36)$ & $(41.35)$ & $(37.50)$ \\
\hline No & 16 & 33 & 49 & 23 & 28 & 51 & 39 & 61 & 100 \\
& $(72.73)$ & $(61.11)$ & $(64.47)$ & $(67.64)$ & $(56.00)$ & $(60.71)$ & $(69.64)$ & $(58.65)$ & $(62.50)$ \\
\hline
\end{tabular}

Note: Figures in the parentheses indicate percentage

Table 6: Distribution of respondents on the basis of belief regarding impact of the cancer $\quad$ (Multiple response).

\begin{tabular}{|c|c|c|c|c|c|c|c|c|c|}
\hline \multirow{2}{*}{$\begin{array}{l}\text { Impact of } \\
\text { cancer }\end{array}$} & \multicolumn{3}{|c|}{$\begin{array}{c}\text { Bathinda } \\
(\mathrm{n} 1=76)\end{array}$} & \multicolumn{3}{|c|}{$\begin{array}{c}\text { Sri Muktsar Sahib } \\
(\mathrm{n} 2=84)\end{array}$} & \multicolumn{3}{|c|}{$\begin{array}{c}\text { South Western Region } \\
(\mathrm{N}=160)\end{array}$} \\
\hline & $\begin{array}{c}\text { Male } \\
(\mathrm{n}=\mathbf{2 2})\end{array}$ & $\begin{array}{c}\text { Female } \\
(n=54)\end{array}$ & $\begin{array}{c}\text { Total } \\
(\mathrm{n}=76)\end{array}$ & $\begin{array}{c}\text { Male } \\
(n=34)\end{array}$ & $\begin{array}{c}\text { Female } \\
(n=50)\end{array}$ & $\begin{array}{c}\text { Total } \\
(\mathrm{n}=84)\end{array}$ & $\begin{array}{c}\text { Male } \\
(n=56)\end{array}$ & $\begin{array}{l}\text { Female } \\
(n=104)\end{array}$ & $\begin{array}{c}\text { Total } \\
(\mathrm{n}=160)\end{array}$ \\
\hline $\begin{array}{l}\text { Incapable } \\
\text { of leading } \\
\text { productive } \\
\text { life }\end{array}$ & $\begin{array}{c}12 \\
(54.55)\end{array}$ & $\begin{array}{c}34 \\
(62.96)\end{array}$ & $\begin{array}{c}46 \\
(60.52)\end{array}$ & $\begin{array}{c}30 \\
(88.24)\end{array}$ & $\begin{array}{c}47 \\
(94.00)\end{array}$ & $\begin{array}{c}77 \\
(91.66)\end{array}$ & $\begin{array}{c}42 \\
(75.00)\end{array}$ & $\begin{array}{c}81 \\
(77.88)\end{array}$ & $\begin{array}{c}123 \\
(76.87)\end{array}$ \\
\hline $\begin{array}{l}\text { Eventuality } \\
\text { of death }\end{array}$ & $\begin{array}{c}18 \\
(81.82)\end{array}$ & $\begin{array}{c}45 \\
(83.33)\end{array}$ & $\begin{array}{c}63 \\
(82.89)\end{array}$ & $\begin{array}{c}33 \\
(97.05)\end{array}$ & $\begin{array}{c}47 \\
(94.00)\end{array}$ & $\begin{array}{c}80 \\
(95.23)\end{array}$ & $\begin{array}{c}51 \\
(91.07)\end{array}$ & $\begin{array}{c}92 \\
(88.46)\end{array}$ & $\begin{array}{c}143 \\
(89.37)\end{array}$ \\
\hline $\begin{array}{l}\text { Changed } \\
\text { social roles }\end{array}$ & $\begin{array}{c}10 \\
(45.45)\end{array}$ & $\begin{array}{c}27 \\
(50.00)\end{array}$ & $\begin{array}{c}37 \\
(48.68)\end{array}$ & $\begin{array}{c}14 \\
(41.17)\end{array}$ & $\begin{array}{c}29 \\
(58.00)\end{array}$ & $\begin{array}{c}43 \\
(51.19)\end{array}$ & $\begin{array}{c}24 \\
(42.85)\end{array}$ & $\begin{array}{c}56 \\
(53.84)\end{array}$ & $\begin{array}{c}80 \\
(50.00)\end{array}$ \\
\hline
\end{tabular}

Note: Figures in the parentheses indicate percentage 
Table 7: Distribution of respondents on the basis of most effective mitigating strategy they adopted.

\begin{tabular}{|c|c|c|c|c|c|c|c|c|c|}
\hline \multirow{2}{*}{$\begin{array}{l}\text { Mitigating } \\
\text { strategy }\end{array}$} & \multicolumn{3}{|c|}{$\begin{array}{c}\text { Bathinda } \\
(\mathrm{n} 1=76)\end{array}$} & \multicolumn{3}{|c|}{ Sri Muktsar Sahib (n2=84) } & \multicolumn{3}{|c|}{$\begin{array}{c}\text { South Western Region } \\
(\mathrm{N}=160)\end{array}$} \\
\hline & $\begin{array}{c}\text { Male } \\
(n=22)\end{array}$ & $\begin{array}{c}\text { Female } \\
(n=54)\end{array}$ & $\begin{array}{c}\text { Total } \\
(n=76)\end{array}$ & $\begin{array}{c}\text { Male } \\
(n=34)\end{array}$ & $\begin{array}{c}\text { Female } \\
(n=50)\end{array}$ & $\begin{array}{c}\text { Total } \\
(n=84)\end{array}$ & $\begin{array}{c}\text { Male } \\
(n=56)\end{array}$ & $\begin{array}{l}\text { Female } \\
(n=104)\end{array}$ & $\begin{array}{c}\text { Total } \\
(\mathrm{n}=\mathbf{1 6 0})\end{array}$ \\
\hline $\begin{array}{l}\text { Support } \\
\text { from family }\end{array}$ & $\begin{array}{c}10 \\
(45.45)\end{array}$ & $\begin{array}{c}10 \\
(18.51)\end{array}$ & $\begin{array}{c}20 \\
(26.31)\end{array}$ & $\begin{array}{c}8 \\
(23.52)\end{array}$ & $\begin{array}{c}15 \\
(30.00) \\
\end{array}$ & $\begin{array}{c}23 \\
(27.38)\end{array}$ & $\begin{array}{c}18 \\
(32.14) \\
\end{array}$ & $\begin{array}{c}25 \\
(24.03) \\
\end{array}$ & $\begin{array}{c}43 \\
(26.87) \\
\end{array}$ \\
\hline $\begin{array}{l}\text { Support } \\
\text { from } \\
\text { spouse }\end{array}$ & $\begin{array}{c}3 \\
(13.63)\end{array}$ & $\begin{array}{c}12 \\
(22.22)\end{array}$ & $\begin{array}{c}15 \\
(19.73)\end{array}$ & $\begin{array}{c}9 \\
(26.47)\end{array}$ & $\begin{array}{c}11 \\
(22.00)\end{array}$ & $\begin{array}{c}20 \\
(23.80)\end{array}$ & $\begin{array}{c}12 \\
(21.42)\end{array}$ & $\begin{array}{c}23 \\
(22.11)\end{array}$ & $\begin{array}{c}35 \\
(21.87)\end{array}$ \\
\hline $\begin{array}{l}\text { Motivated } \\
\text { due to off } \\
\text { springs }\end{array}$ & $\begin{array}{c}2 \\
(9.09)\end{array}$ & $\begin{array}{c}5 \\
(9.25)\end{array}$ & $\begin{array}{c}7 \\
(9.21)\end{array}$ & $\begin{array}{c}2 \\
(5.88)\end{array}$ & $\begin{array}{c}6 \\
(12.00)\end{array}$ & $\begin{array}{c}8 \\
(9.52)\end{array}$ & $\begin{array}{c}4 \\
(7.14)\end{array}$ & $\begin{array}{c}11 \\
(10.57)\end{array}$ & $\begin{array}{c}15 \\
(9.37)\end{array}$ \\
\hline $\begin{array}{l}\text { Faith on } \\
\text { god }\end{array}$ & $\begin{array}{c}4 \\
(18.18) \\
\end{array}$ & $\begin{array}{c}18 \\
(33.33) \\
\end{array}$ & $\begin{array}{c}22 \\
(28.94) \\
\end{array}$ & $\begin{array}{c}7 \\
(20.59) \\
\end{array}$ & $\begin{array}{c}9 \\
(18.00) \\
\end{array}$ & $\begin{array}{c}16 \\
(19.05) \\
\end{array}$ & $\begin{array}{c}11 \\
(19.64) \\
\end{array}$ & $\begin{array}{c}27 \\
(25.96)\end{array}$ & $\begin{array}{c}38 \\
(23.75)\end{array}$ \\
\hline $\begin{array}{l}\text { No } \\
\text { Mitigation }\end{array}$ & $\begin{array}{c}3 \\
(13.63)\end{array}$ & $\begin{array}{c}9 \\
(16.67)\end{array}$ & $\begin{array}{c}12 \\
(15.79)\end{array}$ & $\begin{array}{c}8 \\
(23.52)\end{array}$ & $\begin{array}{c}9 \\
(18.00)\end{array}$ & $\begin{array}{c}17 \\
(20.24)\end{array}$ & $\begin{array}{c}11 \\
(19.64)\end{array}$ & $\begin{array}{c}18 \\
(17.31)\end{array}$ & $\begin{array}{c}29 \\
(18.13)\end{array}$ \\
\hline
\end{tabular}

Note: Figures in the parentheses indicate percentage

Table 8: Social interaction after diagnosis of the disease.

\begin{tabular}{|c|c|c|c|c|c|c|c|c|c|}
\hline \multirow{2}{*}{$\begin{array}{l}\text { Social } \\
\text { Interaction }\end{array}$} & \multicolumn{3}{|c|}{$\begin{array}{c}\text { Bathinda } \\
(\mathrm{n} 1=76)\end{array}$} & \multicolumn{3}{|c|}{$\begin{array}{c}\text { Sri Muktsar Sahib } \\
(\mathrm{n} 2=84)\end{array}$} & \multicolumn{3}{|c|}{$\begin{array}{c}\text { South Western Region } \\
(\mathrm{N}=\mathbf{1 6 0})\end{array}$} \\
\hline & $\begin{array}{c}\text { Male } \\
(n=22)\end{array}$ & $\begin{array}{c}\text { Female } \\
(n=54)\end{array}$ & $\begin{array}{c}\text { Total } \\
(n=76)\end{array}$ & $\begin{array}{c}\text { Male } \\
(n=34)\end{array}$ & $\begin{array}{c}\text { Female } \\
(n=50)\end{array}$ & $\begin{array}{c}\text { Total } \\
(n=84)\end{array}$ & $\begin{array}{c}\text { Male } \\
(n=56)\end{array}$ & $\begin{array}{l}\text { Female } \\
(n=104)\end{array}$ & $\begin{array}{c}\text { Total } \\
(n=160)\end{array}$ \\
\hline Always & $\begin{array}{c}15 \\
(68.18)\end{array}$ & $\begin{array}{c}26 \\
(48.15)\end{array}$ & $\begin{array}{c}41 \\
(53.95)\end{array}$ & $\begin{array}{c}20 \\
(58.82)\end{array}$ & $\begin{array}{c}15 \\
(30.00)\end{array}$ & $\begin{array}{c}35 \\
(41.67)\end{array}$ & $\begin{array}{c}35 \\
(62.50)\end{array}$ & $\begin{array}{c}41 \\
(39.42)\end{array}$ & $\begin{array}{c}76 \\
(47.50)\end{array}$ \\
\hline Less & $\begin{array}{c}2 \\
(9.09) \\
\end{array}$ & $\begin{array}{c}19 \\
(35.19)\end{array}$ & $\begin{array}{c}21 \\
(27.63)\end{array}$ & $\begin{array}{c}8 \\
(23.53)\end{array}$ & $\begin{array}{c}23 \\
(46.00)\end{array}$ & $\begin{array}{c}31 \\
(36.90)\end{array}$ & $\begin{array}{c}10 \\
(10.85)\end{array}$ & $\begin{array}{c}42 \\
(40.38)\end{array}$ & $\begin{array}{c}52 \\
(32.50) \\
\end{array}$ \\
\hline Least & $\begin{array}{c}3 \\
(13.63)\end{array}$ & $\begin{array}{c}8 \\
(14.81) \\
\end{array}$ & $\begin{array}{c}11 \\
(14.47)\end{array}$ & $\begin{array}{c}6 \\
(17.65)\end{array}$ & $\begin{array}{c}12 \\
(24.00)\end{array}$ & $\begin{array}{c}18 \\
(21.43)\end{array}$ & $\begin{array}{c}9 \\
(16.07)\end{array}$ & $\begin{array}{c}20 \\
(19.23)\end{array}$ & $\begin{array}{c}29 \\
(18.12)\end{array}$ \\
\hline Nil & $\begin{array}{c}2 \\
(9.09)\end{array}$ & $\begin{array}{c}1 \\
(1.85)\end{array}$ & $\begin{array}{c}3 \\
(3.95)\end{array}$ & - & - & - & $\begin{array}{c}2 \\
(3.57)\end{array}$ & $\begin{array}{c}1 \\
(0.96)\end{array}$ & $\begin{array}{c}3 \\
(1.87)\end{array}$ \\
\hline
\end{tabular}

Note: Figures in the parentheses indicate percentage

Table 9: Mitigation through contacting others

\begin{tabular}{|c|c|c|c|c|c|c|c|c|c|}
\hline \multirow{2}{*}{$\begin{array}{l}\text { Contacted } \\
\text { others }\end{array}$} & \multicolumn{3}{|c|}{$\begin{array}{l}\text { Bathinda } \\
(\mathrm{n} 1=76)\end{array}$} & \multicolumn{3}{|c|}{ Sri Muktsar Sahib (n2=84) } & \multicolumn{3}{|c|}{$\begin{array}{l}\text { South Western Region } \\
(\mathrm{N}=160)\end{array}$} \\
\hline & $\begin{array}{l}\text { Male } \\
(n=22)\end{array}$ & $\begin{array}{l}\text { Female } \\
(n=54)\end{array}$ & $\begin{array}{l}\text { Total } \\
(n=76)\end{array}$ & $\begin{array}{l}\text { Male } \\
(n=34)\end{array}$ & $\begin{array}{l}\text { Female } \\
(n=50)\end{array}$ & $\begin{array}{l}\text { Total } \\
(n=84)\end{array}$ & $\begin{array}{l}\text { Male } \\
(n=56)\end{array}$ & $\begin{array}{l}\text { Female } \\
(n=104)\end{array}$ & $\begin{array}{l}\text { Total } \\
(n=160)\end{array}$ \\
\hline Yes & $\begin{array}{l}14 \\
(63.63)\end{array}$ & $\begin{array}{l}28 \\
(51.85)\end{array}$ & $\begin{array}{l}42 \\
(55.26) \\
\end{array}$ & $\begin{array}{l}26 \\
(76.47) \\
\end{array}$ & $\begin{array}{l}36 \\
(72.00) \\
\end{array}$ & $\begin{array}{l}62 \\
(73.80) \\
\end{array}$ & $\begin{array}{l}40 \\
(71.42) \\
\end{array}$ & $\begin{array}{l}64 \\
(61.53) \\
\end{array}$ & $\begin{array}{l}104 \\
(65.00)\end{array}$ \\
\hline No & $\begin{array}{l}8 \\
(36.36)\end{array}$ & $\begin{array}{l}26 \\
(48.14)\end{array}$ & $\begin{array}{l}34 \\
(44.73)\end{array}$ & $\begin{array}{l}8 \\
(23.52)\end{array}$ & $\begin{array}{l}14 \\
(28.00)\end{array}$ & $\begin{array}{l}22 \\
(26.19)\end{array}$ & $\begin{array}{l}16 \\
(28.57)\end{array}$ & $\begin{array}{l}40 \\
(38.46)\end{array}$ & $\begin{array}{l}56 \\
(35.00)\end{array}$ \\
\hline $\begin{array}{l}\text { Information } \\
\text { gathered * }\end{array}$ & $\begin{array}{l}\text { Male } \\
(n=14)\end{array}$ & $\begin{array}{l}\text { Female } \\
(n=28)\end{array}$ & $\begin{array}{l}\text { Total } \\
(n=42)\end{array}$ & $\begin{array}{l}\text { Male } \\
(n=26)\end{array}$ & $\begin{array}{r}\text { Female } \\
(n=36)\end{array}$ & $\begin{array}{l}\text { Total } \\
(n=62)\end{array}$ & $\begin{array}{l}\text { Male } \\
(n=40)\end{array}$ & $\begin{array}{l}\text { Female } \\
(n=64)\end{array}$ & $\begin{array}{l}\text { Total } \\
(n=104)\end{array}$ \\
\hline $\begin{array}{l}\text { Prognosis of } \\
\text { cancer }\end{array}$ & $\begin{array}{l}13 \\
(92.85)\end{array}$ & $\begin{array}{l}19 \\
(67.25) \\
\end{array}$ & $\begin{array}{l}32 \\
(76.19)\end{array}$ & $\begin{array}{l}23 \\
(88.46)\end{array}$ & $\begin{array}{l}29 \\
(80.55)\end{array}$ & $\begin{array}{l}52 \\
(83.87)\end{array}$ & $\begin{array}{l}36 \\
(90.00)\end{array}$ & $\begin{array}{l}48 \\
(75.00)\end{array}$ & $\begin{array}{l}84 \\
(80.76)\end{array}$ \\
\hline $\begin{array}{l}\text { Alternative } \\
\text { treatments } \\
\text { available }\end{array}$ & $\begin{array}{l}8 \\
(57.14)\end{array}$ & $\begin{array}{l}23 \\
(82.14)\end{array}$ & $\begin{array}{l}31 \\
(73.80)\end{array}$ & $\begin{array}{l}16 \\
(61.53)\end{array}$ & $\begin{array}{l}29 \\
(80.55)\end{array}$ & $\begin{array}{l}45 \\
(72.58)\end{array}$ & $\begin{array}{l}24 \\
(60.00)\end{array}$ & $\begin{array}{l}52 \\
(81.25)\end{array}$ & $\begin{array}{l}76 \\
(73.07)\end{array}$ \\
\hline
\end{tabular}

Note: Figures in the parentheses indicate percentage

* Multiple Response 
Table 10: Distribution of respondents on the basis of association between beliefs and socio-economic aspects of cancer patients

\begin{tabular}{|l|l|l|}
\hline Parameters & Chi Square & P-Value \\
\hline Stage of cancer & 8.23 & 0.05 \\
\hline Delay in seeking treatment & 36.64 & 0.01 \\
\hline Gender & 8.32 & 0.05 \\
\hline Caste & 14.74 & 0.05 \\
\hline Religion & 5.171 & 0.05 \\
\hline Education & 24.02 & 0.01 \\
\hline
\end{tabular}

cancer. This sense of fear coupled with inadequate knowledge of disease, symptoms and the financial support by the Government, an initiative by them as seem to lead to adoption of a negative attitude towards health care in general (Kishore et al., 2008). Disturbances in menstrual cycle, reduced fertility/ infertility, prolonged time-to-pregnancy, spontaneous abortion, stillbirths and developmental defects have been associated with the frequent exposure to pesticides (Kaur, 2013).

Table 6 reveals that three fourth $(76.8 \%)$ of the respondents from south western region believe that they were incapable of leading a productive life. Specially, the female respondents $(77.8 \%)$ feel more than male respondents $(75.0 \%)$ of the region. In Bathinda, 60.5 per cent of the respondents believe that they were incapable of leading a productive life and 39.4 per cent were capable of leading a productive life. In the study region, it was reported that they had not indulged in sexual as relation with their partner since the treatment was under process which was not a physiological imbalance among majority of the respondents. Many people in the society believe that cancer is incurable and is a fatal disease but the fact is that cancer is the most curable of all chronic diseases Kaur and Sharma 2021). The likelihood of the infection getting to be deadly relied upon the sort and type of disease as well as how best in class it seems to be. If detected at early stage and ensued by medical treatment cancer can be cured. In the present study, beliefs such as fatalism and incurability were found to be associated with cancer. Cancer fatalism is the belief that death is inevitable when cancer is present. Fatalism is traditionally conceived as the perception that events and/or health issues are out of an individual's control. The fatalistic individual expects that the result of the sickness will be negative. Malignancy capitulation to the inevitable is a multidimensional build, which is guided by various factors, for example, mindfulness, recognitions and convictions about the malady (Mayo et al., 2001). The perusal of table 6 indicated that cancer patient believe that they will eventually die. Majority of the respondents $(89.3 \%)$ in the region believe that they will die because of this disease soon. The eventuality was that deaths were more reported in case of males than females. In the comparison of both the district Bathinda and Muktsar, majority $(83.3 \%$ and $94.0 \%)$ of the female respondents believe that cancer patient eventually died because majority of the females in this region were illiterate and believe what others feel about the disease easily. Male respondents $(81.8 \%$ and $97.0 \%)$ of both the district Bathinda and Muktsar believe that cancer patient eventually died. After the diagnosis of cancer and during treatment, patient's life started moving in very different way, so their social life also gets affected due to menace of cancer. Half of the respondents $(50.0 \%)$ had affected effect on their social roles due to the disease.

\section{Mitigating strategies}

The perusal of the table 7 reveals that one fourth of the respondents $(26.8 \%)$ adopted the strategy of support from family followed by having faith in God (23.7\%), got support from spouse (21.8\%). Ten per cent of the respondents got motivated due to off springs for the treatment of this disease and rest 18.1 percent had not adopted any mitigation strategy for this disease.

\section{Social interaction after diagnosis of the disease}

The distribution of the respondents on the basis of interaction after the diagnosis of the disease is presented in the table 8 . The data indicated that in the region, 47.5 per cent of the respondents always used to interact with their family members, friends, relatives, neighbors after they were diagnosed with 
Table 11: Distribution of respondents on the basis oftheir perception on the role of environment factors in causing cancer (Multiple responses).

\begin{tabular}{|c|c|c|c|c|c|c|c|c|c|c|c|c|c|c|c|c|c|c|}
\hline \multirow{3}{*}{$\begin{array}{l}\text { Environmental } \\
\text { Factors }\end{array}$} & \multicolumn{6}{|c|}{ Bathinda(n1=76) } & \multicolumn{6}{|c|}{ Sri Muktsar Sahib(n2=84) } & \multicolumn{6}{|c|}{ South Western Region $(\mathrm{N}=160)$} \\
\hline & \multicolumn{3}{|c|}{ Male(n=22) } & \multicolumn{3}{|c|}{ Female $(n=54)$} & \multicolumn{3}{|c|}{$\operatorname{Male}(n=34)$} & \multicolumn{3}{|c|}{ Female $(n=50)$} & \multicolumn{3}{|c|}{ Male $(n=56)$} & \multicolumn{3}{|c|}{ Female $(n=104)$} \\
\hline & Yes & No & $\begin{array}{l}\text { Cnt } \\
\text { say }\end{array}$ & Yes & No & $\begin{array}{l}\text { Cnt } \\
\text { say }\end{array}$ & Yes & No & $\begin{array}{l}\text { Cnt } \\
\text { say }\end{array}$ & Yes & No & $\begin{array}{l}\text { Cnt } \\
\text { say }\end{array}$ & Yes & No & $\begin{array}{l}\text { Cnt } \\
\text { say }\end{array}$ & Yes & No & $\begin{array}{l}\text { Cnt } \\
\text { say }\end{array}$ \\
\hline $\begin{array}{l}\text { Heavy metal in } \\
\text { groundwater }\end{array}$ & $\begin{array}{l}21 \\
(95.45) \\
\end{array}$ & $\begin{array}{l}1 \\
(4.55)\end{array}$ & - & $\begin{array}{l}54 \\
(100.0) \\
\end{array}$ & - & 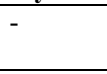 & $\begin{array}{l}34 \\
(100.00) \\
\end{array}$ & - & - & $\begin{array}{l}5 \\
(10.00)\end{array}$ & - & J & $\begin{array}{l}55 \\
(98.21) \\
\end{array}$ & $\begin{array}{l}1 \\
(1.79) \\
\end{array}$ & 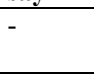 & $\begin{array}{l}59 \\
(56.73)\end{array}$ & - & J \\
\hline $\begin{array}{ll}\text { Due } & \text { to } \\
\text { industrial } & \\
\text { waste } & \\
\end{array}$ & $\begin{array}{l}5 \\
(22.73)\end{array}$ & $\begin{array}{l}13 \\
(59.09)\end{array}$ & $\begin{array}{l}4 \\
(18.18)\end{array}$ & $\begin{array}{l}5 \\
(9.26)\end{array}$ & $\begin{array}{l}30 \\
(55.56)\end{array}$ & $\begin{array}{l}19 \\
(35.19)\end{array}$ & $\begin{array}{l}12 \\
(35.29)\end{array}$ & $\begin{array}{l}14 \\
(41.18)\end{array}$ & $\begin{array}{l}7 \\
(20.59)\end{array}$ & $\begin{array}{l}25 \\
(50.00)\end{array}$ & $\begin{array}{l}12 \\
(24.00)\end{array}$ & $\begin{array}{l}13 \\
(26.00)\end{array}$ & $\begin{array}{l}17 \\
(30.36)\end{array}$ & $\begin{array}{l}27 \\
(48.21\end{array}$ & $\begin{array}{l}11 \\
(19.64)\end{array}$ & $\begin{array}{l}30 \\
(28.85\end{array}$ & $\begin{array}{l}42 \\
(40.38)\end{array}$ & $\begin{array}{l}32 \\
(30.77)\end{array}$ \\
\hline $\begin{array}{l}\text { Ash from } \\
\text { thermal power } \\
\text { plant }\end{array}$ & $\begin{array}{l}15 \\
(68.18)\end{array}$ & $\begin{array}{l}4 \\
(18.18)\end{array}$ & $\begin{array}{l}3 \\
(13.64)\end{array}$ & $\begin{array}{l}35 \\
(64.81)\end{array}$ & $\begin{array}{l}2 \\
(3.70)\end{array}$ & $\begin{array}{l}7 \\
(12.96)\end{array}$ & $\begin{array}{l}17 \\
(50.00)\end{array}$ & $\begin{array}{l}17 \\
(50.00)\end{array}$ & - & $\begin{array}{l}17 \\
(34.00)\end{array}$ & $\begin{array}{l}33 \\
(66.00)\end{array}$ & - & $\begin{array}{l}32 \\
(57.14)\end{array}$ & $\begin{array}{l}21 \\
(37.50)\end{array}$ & $\begin{array}{l}3 \\
(5.36)\end{array}$ & $\begin{array}{l}52 \\
(50.00)\end{array}$ & $\begin{array}{l}35 \\
(33.65)\end{array}$ & $\begin{array}{l}7 \\
(6.73)\end{array}$ \\
\hline $\begin{array}{ll}\text { Gases } & \text { from } \\
\text { refinery } & \\
\end{array}$ & $\begin{array}{l}15 \\
(68.18) \\
\end{array}$ & $\begin{array}{l}5 \\
(22.73) \\
\end{array}$ & $\begin{array}{l}2 \\
(9.09)\end{array}$ & $\begin{array}{l}25 \\
(46.30) \\
\end{array}$ & $\begin{array}{l}27 \\
(50.00)\end{array}$ & $\begin{array}{l}2 \\
(3.70)\end{array}$ & $\begin{array}{l}22 \\
(64.71)\end{array}$ & $\begin{array}{l}12 \\
(35.29)\end{array}$ & - & $\begin{array}{l}25 \\
(50.00)\end{array}$ & $\begin{array}{l}25 \\
(50.00)\end{array}$ & 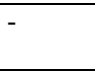 & $\begin{array}{l}37 \\
(66.07)\end{array}$ & $\begin{array}{l}17 \\
(30.36)\end{array}$ & $\begin{array}{l}2 \\
(3.57)\end{array}$ & $\begin{array}{l}50 \\
(48.08)\end{array}$ & $\begin{array}{l}52 \\
(50.00) \\
\end{array}$ & $\begin{array}{l}2 \\
(1.92)\end{array}$ \\
\hline $\begin{array}{l}\text { Use of banned } \\
\text { chemicals }\end{array}$ & $\begin{array}{l}9 \\
(40.91)\end{array}$ & $\begin{array}{l}11 \\
(50.00)\end{array}$ & $\begin{array}{l}2 \\
(9.09)\end{array}$ & $\begin{array}{l}12 \\
(22.22)\end{array}$ & $\begin{array}{l}28 \\
(51.85)\end{array}$ & $\begin{array}{l}13 \\
(24.07)\end{array}$ & $\begin{array}{l}17 \\
(50.00)\end{array}$ & $\begin{array}{l}15 \\
(44.12)\end{array}$ & $\begin{array}{l}2 \\
(5.88)\end{array}$ & $\begin{array}{l}8 \\
(16.00)\end{array}$ & $\begin{array}{l}27 \\
(54.00)\end{array}$ & $\begin{array}{l}15 \\
(30.00)\end{array}$ & $\begin{array}{l}26 \\
(46.43)\end{array}$ & $\begin{array}{l}26 \\
(46.43)\end{array}$ & $\begin{array}{l}4 \\
(7.14)\end{array}$ & $\begin{array}{l}20 \\
(19.23)\end{array}$ & $\begin{array}{l}55 \\
(52.88)\end{array}$ & $\begin{array}{l}28 \\
(26.92)\end{array}$ \\
\hline $\begin{array}{l}\text { Higher dose of } \\
\text { chemicals }\end{array}$ & $\begin{array}{l}7 \\
(31.82)\end{array}$ & $\begin{array}{l}7 \\
(31.82)\end{array}$ & $\begin{array}{l}7 \\
(31.82)\end{array}$ & $\begin{array}{l}18 \\
(33.33) \\
\end{array}$ & $\begin{array}{l}13 \\
(24.07)\end{array}$ & $\begin{array}{l}22 \\
(40.74)\end{array}$ & $\begin{array}{l}25 \\
(73.53) \\
\end{array}$ & $\begin{array}{l}5 \\
(14.71)\end{array}$ & $\begin{array}{l}3 \\
(8.82)\end{array}$ & $\begin{array}{l}17 \\
(34.00)\end{array}$ & $\begin{array}{l}14 \\
(28.00)\end{array}$ & $\begin{array}{l}19 \\
(38.00)\end{array}$ & $\begin{array}{l}32 \\
(57.14)\end{array}$ & $\begin{array}{l}12 \\
(21.43)\end{array}$ & $\begin{array}{l}10 \\
(17.86)\end{array}$ & $\begin{array}{l}35 \\
(33.65)\end{array}$ & $\begin{array}{l}27 \\
(25.96)\end{array}$ & $\begin{array}{l}41 \\
(39.42)\end{array}$ \\
\hline $\begin{array}{l}\text { Stubble } \\
\text { burning }\end{array}$ & $\begin{array}{l}18 \\
(81.82) \\
\end{array}$ & $\begin{array}{l}2 \\
(9.09) \\
\end{array}$ & $\begin{array}{l}2 \\
(9.09)\end{array}$ & $\begin{array}{l}47 \\
(87.04)\end{array}$ & $\begin{array}{l}3 \\
(5.56)\end{array}$ & $\begin{array}{l}4 \\
(7.41) \\
\end{array}$ & $\begin{array}{l}27 \\
(79.41)\end{array}$ & $\begin{array}{l}7 \\
(20.59) \\
\end{array}$ & - & $\begin{array}{l}37 \\
(74.00)\end{array}$ & $\begin{array}{l}11 \\
(22.00)\end{array}$ & $\begin{array}{l}2 \\
(4.00)\end{array}$ & $\begin{array}{l}45 \\
(80.36)\end{array}$ & $\begin{array}{l}9 \\
(16.07)\end{array}$ & $\begin{array}{l}2 \\
(3.57) \\
\end{array}$ & $\begin{array}{l}84 \\
(80.77) \\
\end{array}$ & $\begin{array}{l}14 \\
(13.46)\end{array}$ & $\begin{array}{l}6 \\
(5.77) \\
\end{array}$ \\
\hline
\end{tabular}

Note: Figures in the parentheses indicate percentage

Table 12: Distribution of respondents on the basis of their perception regarding role of changing lifestyle in causing cancer (Multiple response).

\begin{tabular}{|c|c|c|c|c|c|c|c|c|c|c|c|c|c|c|c|c|c|c|}
\hline \multirow{3}{*}{ Lifestyle } & \multicolumn{6}{|c|}{ Bathinda(n1=76) } & \multicolumn{6}{|c|}{ Sri Muktsar Sahib (n2=84) } & \multicolumn{6}{|c|}{ South Western Region $(\mathrm{N}=160)$} \\
\hline & \multicolumn{3}{|c|}{ Male(n=22) } & \multicolumn{3}{|c|}{ Female(n=54) } & \multicolumn{3}{|c|}{ Male(n=34) } & \multicolumn{3}{|c|}{ Female(n=50) } & \multicolumn{3}{|c|}{ Male(n=56) } & \multicolumn{3}{|c|}{ Female(n=104) } \\
\hline & Yes & No & $\begin{array}{l}\text { Cnt } \\
\text { say }\end{array}$ & Yes & No & $\begin{array}{l}\text { Cnt } \\
\text { say }\end{array}$ & Yes & No & $\begin{array}{l}\text { Cnt } \\
\text { say }\end{array}$ & Yes & No & $\begin{array}{l}\text { Cnt } \\
\text { say }\end{array}$ & Yes & No & $\begin{array}{l}\text { Cnt } \\
\text { say }\end{array}$ & Yes & No & $\begin{array}{l}\text { Cnt } \\
\text { say }\end{array}$ \\
\hline $\begin{array}{l}\text { Imbalanced } \\
\text { Diet }\end{array}$ & $\begin{array}{l}2 \\
(9.09)\end{array}$ & $\begin{array}{l}3 \\
(13.64)\end{array}$ & $\begin{array}{l}17 \\
(77.27) \\
\end{array}$ & $\begin{array}{l}7 \\
(12.96) \\
\end{array}$ & $\begin{array}{l}11 \\
(20.37) \\
\end{array}$ & $\begin{array}{l}36 \\
(66.67)\end{array}$ & $\begin{array}{l}17 \\
(50.00)\end{array}$ & $\begin{array}{l}10 \\
(29.41) \\
\end{array}$ & $\begin{array}{l}7 \\
(20.59) \\
\end{array}$ & $\begin{array}{l}23 \\
(46.00)\end{array}$ & $\begin{array}{l}9 \\
(18.00)\end{array}$ & $\begin{array}{l}18 \\
(36.00) \\
\end{array}$ & $\begin{array}{l}19 \\
(33.93) \\
\end{array}$ & $\begin{array}{l}13 \\
(23.21) \\
\end{array}$ & $\begin{array}{l}24 \\
(42.86) \\
\end{array}$ & $\begin{array}{l}30 \\
(28.85)\end{array}$ & $\begin{array}{l}20 \\
(19.23) \\
\end{array}$ & $\begin{array}{l}54 \\
(51.92)\end{array}$ \\
\hline $\begin{array}{l}\text { Fast food } \\
\text { culture }\end{array}$ & $\begin{array}{l}1 \\
(4.55)\end{array}$ & $\begin{array}{l}17 \\
(77.27)\end{array}$ & $\begin{array}{l}4 \\
(18.18)\end{array}$ & $\begin{array}{l}2 \\
(3.70)\end{array}$ & $\begin{array}{l}51 \\
(94.44)\end{array}$ & $\begin{array}{l}1 \\
(1.85)\end{array}$ & $\begin{array}{l}2 \\
((5.88)\end{array}$ & $\begin{array}{l}32 \\
(94.12)\end{array}$ & - & $\begin{array}{l}3 \\
(6.00)\end{array}$ & $\begin{array}{l}47 \\
(94.00)\end{array}$ & $\begin{array}{l}1 \\
(2.00)\end{array}$ & $\begin{array}{l}3 \\
(5.36)\end{array}$ & $\begin{array}{l}49 \\
(87.50)\end{array}$ & $\begin{array}{l}4 \\
(7.14)\end{array}$ & $\begin{array}{l}5 \\
(4.81) \\
\end{array}$ & $\begin{array}{l}98 \\
(94.23) \\
\end{array}$ & $\begin{array}{l}2 \\
(1.92)\end{array}$ \\
\hline $\begin{array}{l}\text { Substance } \\
\text { abuse }\end{array}$ & $\begin{array}{l}11 \\
(50.00)\end{array}$ & $\begin{array}{l}11 \\
(50.00)\end{array}$ & - & $\begin{array}{l}5 \\
(9.25)\end{array}$ & $\begin{array}{l}49 \\
(90.74)\end{array}$ & - & $\begin{array}{l}18 \\
(52.94)\end{array}$ & $\begin{array}{l}15 \\
(44.12) \\
\end{array}$ & - & $\begin{array}{l}2 \\
(4.00)\end{array}$ & $\begin{array}{l}48 \\
(96.00)\end{array}$ & - & $\begin{array}{l}29 \\
(51.79)\end{array}$ & $\begin{array}{l}26 \\
(46.43)\end{array}$ & - & $\begin{array}{l}97 \\
(93.26)\end{array}$ & $\begin{array}{l}7 \\
(6.73)\end{array}$ & - \\
\hline $\begin{array}{l}\text { Sedentary } \\
\text { lifestyle }\end{array}$ & $\begin{array}{l}4 \\
(18.18) \\
\end{array}$ & $\begin{array}{l}8 \\
(36.36) \\
\end{array}$ & $\begin{array}{l}10 \\
(45.45) \\
\end{array}$ & $\begin{array}{l}16 \\
(29.63)\end{array}$ & $\begin{array}{l}8 \\
(14.81) \\
\end{array}$ & $\begin{array}{l}30 \\
(55.56)\end{array}$ & $\begin{array}{l}17 \\
(50.00)\end{array}$ & $\begin{array}{l}6 \\
(17.65) \\
\end{array}$ & $\begin{array}{l}6 \\
17.65) \\
\end{array}$ & $\begin{array}{l}25 \\
(50.00) \\
\end{array}$ & $\begin{array}{l}7 \\
(14.00) \\
\end{array}$ & $\begin{array}{l}18 \\
(36.00) \\
\end{array}$ & $\begin{array}{l}21 \\
(37.50) \\
\end{array}$ & $\begin{array}{l}14 \\
(25.00) \\
\end{array}$ & $\begin{array}{l}16 \\
(28.57) \\
\end{array}$ & $\begin{array}{l}41 \\
(39.42) \\
\end{array}$ & $\begin{array}{l}15 \\
(14.42) \\
\end{array}$ & $\begin{array}{l}48 \\
(46.15) \\
\end{array}$ \\
\hline
\end{tabular}

Note: Figures in the parentheses indicate percentage 
Table 13: Distribution of respondents on the basis of their perception regarding role of changing in daily practices in causing cancer (Multiple response).

\begin{tabular}{|c|c|c|c|c|c|c|c|c|c|c|c|c|c|c|c|c|c|c|}
\hline \multirow{3}{*}{ Daily practices } & \multicolumn{6}{|c|}{ Bathinda(n1=76) } & \multicolumn{6}{|c|}{ Sri Muktsar Sahib (n2=84) } & \multicolumn{6}{|c|}{ South Western Region $(\mathrm{N}=160)$} \\
\hline & \multicolumn{3}{|c|}{ Male(n=22) } & \multicolumn{3}{|c|}{ Female(n=54) } & \multicolumn{3}{|c|}{ Male(n=34) } & \multicolumn{3}{|c|}{ Female(n=50) } & \multicolumn{3}{|c|}{ Male(n=56) } & \multicolumn{3}{|c|}{ Female $(n=104)$} \\
\hline & Yes & No & $\begin{array}{l}\text { Cnt } \\
\text { say }\end{array}$ & Yes & No & $\begin{array}{l}\text { Cnt } \\
\text { say }\end{array}$ & Yes & No & $\begin{array}{l}\text { Cnt } \\
\text { say }\end{array}$ & Yes & No & $\begin{array}{l}\text { Cnt } \\
\text { say }\end{array}$ & Yes & No & $\begin{array}{l}\text { Cnt } \\
\text { say }\end{array}$ & Yes & No & $\begin{array}{l}\text { Cnt } \\
\text { say }\end{array}$ \\
\hline $\begin{array}{ll}\text { Reuse of } & \text { cold } \\
\text { drinks/juice/Bisleri } & \\
\text { bottles } & \\
\end{array}$ & $\begin{array}{l}19 \\
(86.36)\end{array}$ & $\begin{array}{l}3 \\
(13.64)\end{array}$ & - & $\begin{array}{l}52 \\
(96.30)\end{array}$ & $\begin{array}{l}2 \\
(3.70)\end{array}$ & $\begin{array}{l}1 \\
(1.85)\end{array}$ & $\begin{array}{l}27 \\
(79.41)\end{array}$ & $\begin{array}{l}7 \\
(20.59)\end{array}$ & - & $\begin{array}{l}42 \\
(84.00)\end{array}$ & $\begin{array}{l}8 \\
(16.00)\end{array}$ & - & $\begin{array}{l}46 \\
(82.14)\end{array}$ & $\begin{array}{l}10 \\
(17.86)\end{array}$ & - & $\begin{array}{l}94 \\
(90.38)\end{array}$ & $\begin{array}{l}10 \\
(9.62)\end{array}$ & $\begin{array}{l}1 \\
(0.96)\end{array}$ \\
\hline $\begin{array}{l}\text { Reuse of paint buckets or } \\
\text { pesticides /insecticides } \\
\text { bottles for storing food } \\
\text { items }\end{array}$ & $\begin{array}{l}16 \\
(72.73)\end{array}$ & $\begin{array}{l}6 \\
(27.27)\end{array}$ & - & $\begin{array}{l}45 \\
(83.33)\end{array}$ & $\begin{array}{l}9 \\
(16.67)\end{array}$ & - & $\begin{array}{l}22 \\
(64.71)\end{array}$ & $\begin{array}{l}12 \\
(35.29)\end{array}$ & - & $\begin{array}{l}34 \\
(68.00)\end{array}$ & $\begin{array}{l}16 \\
(32.00)\end{array}$ & - & $\begin{array}{l}38 \\
(67.86)\end{array}$ & $\begin{array}{l}18 \\
(32.14)\end{array}$ & - & $\begin{array}{l}79 \\
(75.96)\end{array}$ & $\begin{array}{l}25 \\
(24.04)\end{array}$ & - \\
\hline $\begin{array}{l}\text { Use of untreated canal } \\
\text { water }\end{array}$ & $\begin{array}{l}6 \\
(27.27)\end{array}$ & $\begin{array}{l}15 \\
(68.18)\end{array}$ & $\begin{array}{l}1 \\
(4.55)\end{array}$ & $\begin{array}{l}13 \\
(24.07)\end{array}$ & $\begin{array}{l}40 \\
(74.07)\end{array}$ & $\begin{array}{l}1 \\
(1.85)\end{array}$ & - & $\begin{array}{l}34 \\
(100.00)\end{array}$ & - & - & $\begin{array}{l}50 \\
(100.00) \\
\end{array}$ & - & $\begin{array}{l}6 \\
(10.71)\end{array}$ & $\begin{array}{l}49 \\
(87.50)\end{array}$ & $\begin{array}{l}1 \\
(1.79)\end{array}$ & $\begin{array}{l}13 \\
(12.50)\end{array}$ & $\begin{array}{l}90 \\
(86.54)\end{array}$ & $\begin{array}{l}1 \\
(0.96)\end{array}$ \\
\hline Brackish ground water & $\begin{array}{l}3 \\
(13.64)\end{array}$ & $\begin{array}{l}19 \\
(86.36)\end{array}$ & - & $\begin{array}{l}5 \\
(9.26)\end{array}$ & $\begin{array}{l}48 \\
(88.89)\end{array}$ & $\begin{array}{l}1 \\
(1.85)\end{array}$ & - & $\begin{array}{l}34 \\
(100.00)\end{array}$ & - & - & $\begin{array}{l}50 \\
(100.00)\end{array}$ & - & $\begin{array}{l}3 \\
(5.36)\end{array}$ & $\begin{array}{l}53 \\
(94.64)\end{array}$ & - & $\begin{array}{l}5 \\
(4.81)\end{array}$ & $\begin{array}{l}98 \\
(94.23)\end{array}$ & $\begin{array}{l}1 \\
(0.96)\end{array}$ \\
\hline $\begin{array}{l}\text { Reuse of disposables } \\
\text { plastic containers }\end{array}$ & $\begin{array}{l}7 \\
(31.82)\end{array}$ & $\begin{array}{l}15 \\
(68.18)\end{array}$ & - & $\begin{array}{l}26 \\
(48.15)\end{array}$ & $\begin{array}{l}27 \\
(50.00)\end{array}$ & $\begin{array}{l}1 \\
(1.85)\end{array}$ & $\begin{array}{l}20 \\
(58.82)\end{array}$ & $\begin{array}{l}14 \\
(41.18)\end{array}$ & - & $\begin{array}{l}21 \\
(42.00)\end{array}$ & $\begin{array}{l}29 \\
(58.00)\end{array}$ & - & $\begin{array}{l}27 \\
(48.21)\end{array}$ & $\begin{array}{l}29 \\
(51.79)\end{array}$ & - & $\begin{array}{l}47 \\
(45.19)\end{array}$ & $\begin{array}{l}56 \\
(53.85)\end{array}$ & $\begin{array}{l}1 \\
(0.96)\end{array}$ \\
\hline $\begin{array}{l}\text { Use of newspaper to } \\
\text { wrap chapattis }\end{array}$ & $\begin{array}{l}16 \\
(72.73)\end{array}$ & $\begin{array}{l}6 \\
(27.27) \\
\end{array}$ & - & $\begin{array}{l}32 \\
(59.26) \\
\end{array}$ & $\begin{array}{l}22 \\
(40.74)\end{array}$ & - & $\begin{array}{l}18 \\
(52.94)\end{array}$ & $\begin{array}{l}16 \\
(47.06)\end{array}$ & - & $\begin{array}{l}28 \\
(56.00) \\
\end{array}$ & $\begin{array}{l}20 \\
(40.00)\end{array}$ & - & $\begin{array}{l}34 \\
(60.71)\end{array}$ & $\begin{array}{l}22 \\
(39.29) \\
\end{array}$ & -- & $\begin{array}{l}60 \\
(57.69) \\
\end{array}$ & $\begin{array}{l}42 \\
(40.38)\end{array}$ & - \\
\hline Use of tandoori food & $\begin{array}{l}8 \\
(36.36) \\
\end{array}$ & $\begin{array}{l}13 \\
(59.09) \\
\end{array}$ & $\begin{array}{l}1 \\
(4.55) \\
\end{array}$ & $\begin{array}{l}28 \\
(51.85) \\
\end{array}$ & $\begin{array}{l}26 \\
(48.15) \\
\end{array}$ & - & $\begin{array}{l}1 \\
(2.94) \\
\end{array}$ & $\begin{array}{l}33 \\
(97.06) \\
\end{array}$ & - & $\begin{array}{l}4 \\
(8.00) \\
\end{array}$ & $\begin{array}{l}46 \\
(92.00) \\
\end{array}$ & - & $\begin{array}{l}9 \\
(16.07) \\
\end{array}$ & $\begin{array}{l}46 \\
(82.14)\end{array}$ & $\begin{array}{l}1 \\
(1.79)\end{array}$ & $\begin{array}{l}32 \\
(30.77) \\
\end{array}$ & $\begin{array}{l}72 \\
(69.23) \\
\end{array}$ & - \\
\hline
\end{tabular}

Note: Figures in the parentheses indicate percentage

Table 14: $\quad$ Perception regarding curability of cancer.

\begin{tabular}{|c|c|c|c|c|c|c|c|c|c|}
\hline \multirow[b]{2}{*}{ Perceptions } & \multicolumn{3}{|c|}{ Bathinda $(\mathrm{n} 1=76)$} & \multicolumn{3}{|c|}{ Sri Muktsar Sahib (n2=84) } & \multicolumn{3}{|c|}{ South Western Region $(\mathrm{N}=160)$} \\
\hline & $\begin{array}{l}\text { Male } \\
(\mathrm{n}=22)\end{array}$ & $\begin{array}{l}\text { Female } \\
(\mathrm{n}=54)\end{array}$ & $\begin{array}{l}\text { Total } \\
(\mathrm{n}=76)\end{array}$ & $\begin{array}{l}\text { Male } \\
(\mathrm{n}=34)\end{array}$ & $\begin{array}{l}\text { Female } \\
(\mathrm{n}=50)\end{array}$ & $\begin{array}{l}\text { Total } \\
(\mathrm{n}=84)\end{array}$ & $\begin{array}{l}\text { Male } \\
(\mathrm{n}=56)\end{array}$ & \begin{tabular}{|l}
$\begin{array}{l}\text { Female } \\
(\mathrm{n}=104)\end{array}$ \\
\end{tabular} & $\begin{array}{l}\text { Total } \\
(\mathrm{n}=160)\end{array}$ \\
\hline Curable & $\begin{array}{l}8 \\
(36.36)\end{array}$ & $\begin{array}{l}23 \\
(42.59)\end{array}$ & $\begin{array}{l}31 \\
(40.78)\end{array}$ & $\begin{array}{l}5 \\
(14.70)\end{array}$ & $\begin{array}{l}14 \\
(28.00)\end{array}$ & $\begin{array}{l}19 \\
(22.61)\end{array}$ & $\begin{array}{l}13 \\
(23.21)\end{array}$ & \begin{tabular}{|l}
37 \\
$(35.57)$
\end{tabular} & $\begin{array}{l}50 \\
(31.25)\end{array}$ \\
\hline $\begin{array}{l}\text { Non } \\
\text { Curable } \\
\end{array}$ & $\begin{array}{l}14 \\
(63.64)\end{array}$ & $\begin{array}{l}31 \\
(57.41)\end{array}$ & $\begin{array}{l}45 \\
(59.21) \\
\end{array}$ & $\begin{array}{l}29 \\
(85.29)\end{array}$ & $\begin{array}{l}36 \\
(72.00)\end{array}$ & $\begin{array}{l}65 \\
(77.38) \\
\end{array}$ & $\begin{array}{l}43 \\
(76.78)\end{array}$ & \begin{tabular}{|l|}
67 \\
$(64.42)$ \\
\end{tabular} & $\begin{array}{l}110 \\
(68.75) \\
\end{array}$ \\
\hline
\end{tabular}

Note: Figures in the parentheses indicate percentage 
the disease. Interacting with different type of peoples make them free from negative thoughts. One third $(32.5 \%)$ of the respondents used to interact less with their family members, their friends, relatives, neighbors after they were diagnosed with the disease. Eighteen per cent of the respondents interact least with their family members, their friends, relatives, neighbors after they were diagnosed with this disease. Only three respondents did not want to interact with anyone. They just want to live in a calm, peaceful and isolated place without any tension and interference of others. Overall, majority of the respondents used to interact with their family and acquaintances.

\section{Mitigation through contacting others}

Usually cancer patient try to contact another cancer patient to know about the course and alternative treatment. The perusal of Table 9 highlights that two third $(65.0 \%)$ of the respondents try to contact another cancer patient to know about the prognosis of the disease and one third (35.0\%) of the respondent did not want to contact any cancer patient to know any outcome of the disease. In Bathinda district, the females (48.1\%) respondents did not try to contact any cancer patient nearby them as compared to females $(28.0 \%)$ respondents of Muktsar district respectively. More than three fourth $(80.7 \%)$ of the respondents want to contact cancer patient to know about prognosis of cancer in which males $(90.0 \%)$ were more interested to contact cancer patient than their female counterparts $(75.0 \%)$ in the study region. Eight one percent of the female respondents contacted cancer patient to know about the alternative treatment of cancer their male counterparts $(60.0 \%)$ in the region.

Association between beliefs and socio-economic aspects of cancer patients

The perusal of Table 10 highlights the association between beliefs and socio-economic aspects of cancer patients. It was seen by scoring the all beliefs and socio -economic parameters like stage of cancer, delay in seeking treatment, gender, caste, religion and education. With the cube root method, the association was categorized into low, medium and high. The association between beliefs and socio-economic aspects of cancer patients were highly significant in stage of cancer with chi square value 8.23 , followed by delay in seeking treatment
(36.64), gender (8.32), caste (14.74), religion (5.17) and education (24.02).

\section{Perceptions}

Perception is someone's ability to notice and understand the things that are not obvious to other people. The quality of being aware of things through the physical senses. Respondent's perception on the role of environment factors. Environmental factors or environment pollution plays a significant casual factor in spreading the disease. It was probed from the respondents and data given in table 11 indicated that almost all respondents claimed that there was heavy metal present in the underground water. Sixty-sixper cent of the respondents blamed gases omitted by the Guru Gobind Singh refinery situated at Kanakwal village in the Talwandisabo block as the prime cause of environment pollution and factor behind widespread of cancer. More than 50 per cent said that ash omitted from thermal power plants too had a role behind the disease. In this region two thermal power plants were situation one in Bathinda city (Guru Nanak Dev Thermal Power Plant, Bathinda) and another at Banawala village, Talwandisabo (Talwandi Sabo Power Limited). Forty six per cent respondents were blamed for the use of banned chemicals on crops and more than 80 percent of the respondents held for burning the stubble, as a major cause behind the disease.

\section{Respondent's perception regarding role of change in lifestyle}

The distribution of respondents on the basis of their perceptions regarding role of change in lifestyle is shown in table 12. One third of the male respondents perceived imbalanced diet and sedentary lifestyle behind as a reason causing cancer and half of the male respondents and significantly majority of the female respondents (93.3\%) perceived substance abuse as a reason behind causing cancer in the region. However, fast food culture was not perceived as a threat behind causing cancer by the majority of the respondents.

\section{Basis of their perception regarding role of change in daily practices}

The distribution of respondents on the basis of their perceptions regarding role of change in daily practices is shown in the Table 13. The data reveal 
that majority of the respondents $(90.0 \%$ and $82.3 \%)$ female and male perceived use of cold drinks/juice/ bisleri bottles. Ten per cent of the respondents perceived brackish ground water as a cause behind causing cancer. Half of the respondents $(48.2 \%)$ perceived disposable utensils as a reason behind the cause cancer in the region. Nearly two third $(61.2 \%)$ of the respondents perceived use of newspaper to wrap rotis/ chapaties as a reason behind causing cancer in the region.

\section{Perception regarding curability of cancer}

Table 14 indicated that in the study majority $(68.7 \%)$ of the respondent's didn't believe that cancer patient will be cured because majority of the respondents in this region were illiterate and believed in indigenous methods of treatment. They believe that percentage of survival of cancer patients was less as compared to patients of other diseases. Just 31.25 per cent of the respondents believed that cancer patient will be cured because of advancement in medical sciences for the treatment. The respondents got more aware about the disease and started getting the proper checkups from the qualified doctors rather than the traditional healers.

\section{References}

Beeken, R. J., Simon, A. E., von Wagner, C., Whitaker, K. L., \& Wardle, J. (2011). Cancer fatalism: deterring early presentation and increasing social inequalities?. Cancer Epidemiology and Prevention Biomarkers, 20(10), 2127. 2131.

Chataut, R., Pandey, A., \& Rao, N. S. (2015). Treatment seeking behaviour and cost of care among cancer patients in Nepal. Hindu, 79, 79.

Corrigan, P.W. (2014).The Stigma of Disease and Disability: Understanding Causes and Overcoming Injustices. Washington, DC, American Psychological Association, pp: 165 .

Daher, M. (2012). Cultural beliefs and values in cancer patients. Annals of oncology, 23, iii66-iii69.

Katz, I., Hass, R. G., Parisi, N., Astone, J., McEvaddy, D., \& Lucido, D. J. (1987). Lay people's and health care personnel's perceptions of cancer, AIDS, cardiac, and diabetic patients. Psychological reports, 60(2), 615-629.

Kaur, L., \& Sharma, S. (2019). Socio-Economic and psychological consequence of cancer from patient's perspective in South-

\section{Conclusion}

It is pertinent to correct false notions and unscientific beliefs held by the society in order to save upon the precious human resource which might get wasted due to dilly dallying the actions required to mitigate the situation. People need to be sensitized regarding the judicious use of natural resources to promote sustainable development and to minimize the risk caused by unmindful human endeavor. Unprecedented use of chemicals should be monitored through government agencies and guilty should be punished. NGT (National Green Tribunal) should strictly enforce their pollution control measures in order to get rid of pollution caused by industrial units. Civil Society Organizations could act as a watchdog to the voice against any unlawful complaisancybetween MNCs, bureaucrats and the policy planners.

\section{Acknowledgement}

The authors are thankful to the residents of various villages of south western Punjab who made authors a part of their family and share their information.

Western Punjab. Journal of Pharmo and Photochemical, 4, SP4, 116-122.

Kaur, L., \& Sharma, S. (2020). Quality of Health Services and State Intervention for cancer in South Western Region of Punjab. Journal of Community Mobilization and Sustainable Development, 15(2), 339-346.

Kaur, L., \& Sharma, S. (2021). Treatment seeking behavior of cancer patients in south-western Punjab. Journal of Agricultural Development and Policy, 30(2), 101-108.

Kaur, R. (2013). An anthropological study of socio-cultural dimensions and health care of cancer patients with special reference to Malwa region of Punjab. Ph.D. dissertation. Panjab University, Chandigarh, India.

Kaur, R. (2015). Cultural beliefs, cancer and stigma: experiencesof patients from Punjab, India. Ethno medicine, (9) 247-54.

Kishore, J., Ahmad, I., Kaur, R., \& Mohanta, P. K. (2008). Beliefs and perceptions about cancers among patients attending radiotherapy OPD in Delhi, India. Asian Pac $J$ Cancer Prev, 9(1), 155-158. 
Knapp, S., Marziliano, A., \& Moyer, A. (2014). Identity threat and stigma in cancer patients. Health psychology open, 1(1), 2055102914552281.

rural elderly women. Journal of women \& aging, 13(1), 57-72.

Pahwa, M., Babu, N., \& Bhatnagar, S. (2005). Fighting cancer is half the battle...living life is the other half. Journal of Cancer Research and Therapeutics, (1), 98- 102.

Pargement, K. (1997). The psychology of religion and coping. The Guilford Press, New York.

Rai, A., Pradhan, S., Mishra, C. P., Kumar, A., \& Singh, T. B (2014). Health beliefs of women suffering from cancer: A hospital based study. Indian Journal of Prevention and Social Medince, (45), 1-2.
Mayo, R. M., Ureda, J. R., \& Parker, V. G. (2001). Importance of fatalism in understanding mammography screening in

Rangnekar, S. (2012). Gearing up for a karma revolution. The Times of India (English daily newspaper) August 24, pp: 12.

Robb, K. A., Simon, A. E., Miles, A., \& Wardle, J. (2014). Public perceptions of cancer: A qualitative study of the balance of positive and negative beliefs. BMJ Open 4:e005434.

World Health Organization, (2012). GLOBOCAN, Estimated Cancer Incidence, Mortality and Prevalence Worldwide in 2012. Lyon, France, International Agency for Research on Cancer. http://globocan.iarc.fr/pages/fact_sheets_cancer. Aspx. 